\title{
Electrolyte Disorders as Triggers for Takotsubo Cardiomyopathy
}

\author{
Fabio Andreozzi ${ }^{1}$, Giovanni Cuminetti ${ }^{2}$, Rafik Karmali ${ }^{1}$, Prochore Kamgang ${ }^{1}$ \\ ${ }^{1}$ Internal Medicine Department, Brugmann University Hospital, Brussels, Belgium \\ ${ }^{2}$ Cardiology Department, Brugmann University Hospital, Brussels, Belgium
}

How to cite this article: Andreozzi F, Cuminetti G, Karmali R, Kamgang P. Electrolyte disorders as triggers for Takotsubo cardiomyopathy. EJCRIM 2018;5: doi:10.12890/2018_000760.

Conflicts of Interests: The Authors declare that there are no competing interests.

This article is licensed under a Commons Attribution Non-Commercial 4.0 License

\section{ABSTRACT}

A 56-year-old woman presented with cognitive impairment, confusion and slowed speech, muscle cramps and peripheral paraesthesia preceded by vomiting. Blood tests revealed severe hypokalaemia, hyponatremia, hypomagnesemia and hypocalcaemia. Following a diagnosis of Takotsubo cardiomyopathy based on ultrasonography, the patient was treated with electrolyte supplementation and recovered within $48 \mathrm{~h}$. When heart failure is suspected, electrolyte abnormalities should be carefully ruled out as they can affect cardiac function.

\section{LEARNING POINTS}

- The association between electrolyte abnormalities and Takotsubo cardiomyopathy has still not been well established in the literature.

- Hypomagnesemia and hypocalcaemia can contribute to cardiac akinesia and so should be ruled out in heart failure.

- Correction of hypomagnesemia and hypocalcaemia is an important and an under-estimated part of the optimal treatment of cardiac failure.

\section{KEYWORDS}

Takotsubo cardiomyopathy, hypomagnesemia, hypocalcaemia, heart failure

\section{CASE DESCRIPTION}

A 56-year-old woman was admitted to the emergency department because of cognitive impairment, confusion and slowed speech, muscle cramps and peripheral paraesthesia lasting a few days. The patient's family reported very poor food intake in preceding months, and some episodes of vomiting after eating during the previous few days.

The patient's medical history was negative for both somatic and psychiatric disorders. The only medication she was taking was a PPI. She had been smoking cigarettes since adolescence (40 pack-years).

Blood tests revealed severe hypokalaemia $(1.7 \mathrm{mmol} / \mathrm{l})$, hyponatremia $(120 \mathrm{mmol} / \mathrm{l})$, hypomagnesemia $(0.35 \mathrm{mmol} / \mathrm{l})$ and hypocalcaemia (ionized calcium $0.80 \mathrm{mmol} / \mathrm{l}$ ), so the patient was admitted to the intensive care department for electrolyte supplementation. Complete neurological recovery was achieved in $48 \mathrm{~h}$ after partial correction of electrolyte anomalies.

During the following days, we treated progressive cardiac failure with non-invasive ventilation and the administration of diuretics. 


\section{METHODS AND PROCEDURES}

A cardiac ECG revealed diffuse negative T waves in the precordial and lateral leads without chest pain (Fig. 1). Troponin T HS was raised (417 $\mathrm{ng} / \mathrm{l}$, normal value <15) and proBNP was $35,000 \mathrm{pg} / \mathrm{ml}$ (normal value $<125 \mathrm{pg} / \mathrm{ml}$ ). Cardiac echography showed diffuse septo-apical akinesia with an ejection fraction of 25-30\% (Fig. 2).

A diagnosis of Takotsubo cardiomyopathy (TC) was made based on ultrasonography results and confirmed by the finding of no significant lesions in the coronary arteries at coronarography (Fig.3 a-b) A second heart ultrasonography, 10 days later, showed global improvement in ventricular function (FEVG 55\%) and disappearance of the diffuse akinesia.

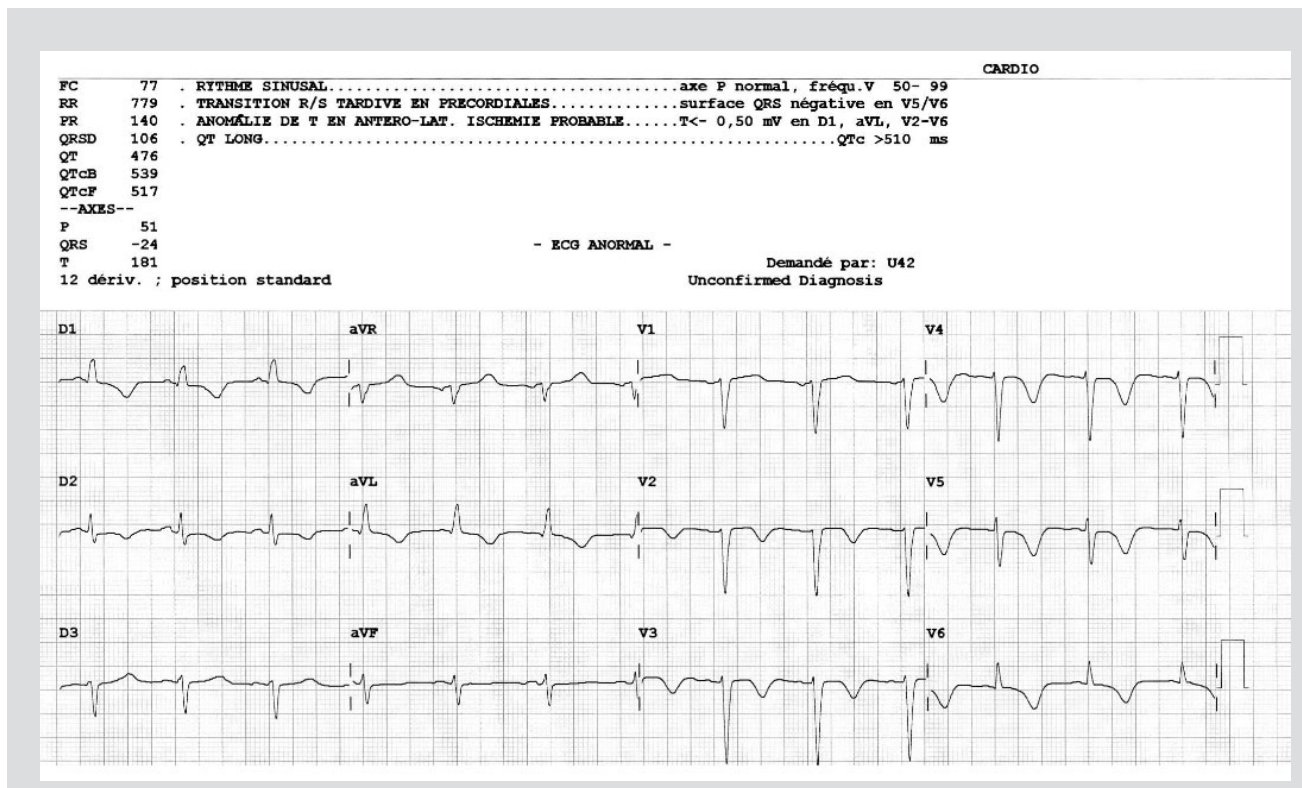

Figure 1. ECG showing diffuse negative T waves in the precordial and lateral leads.

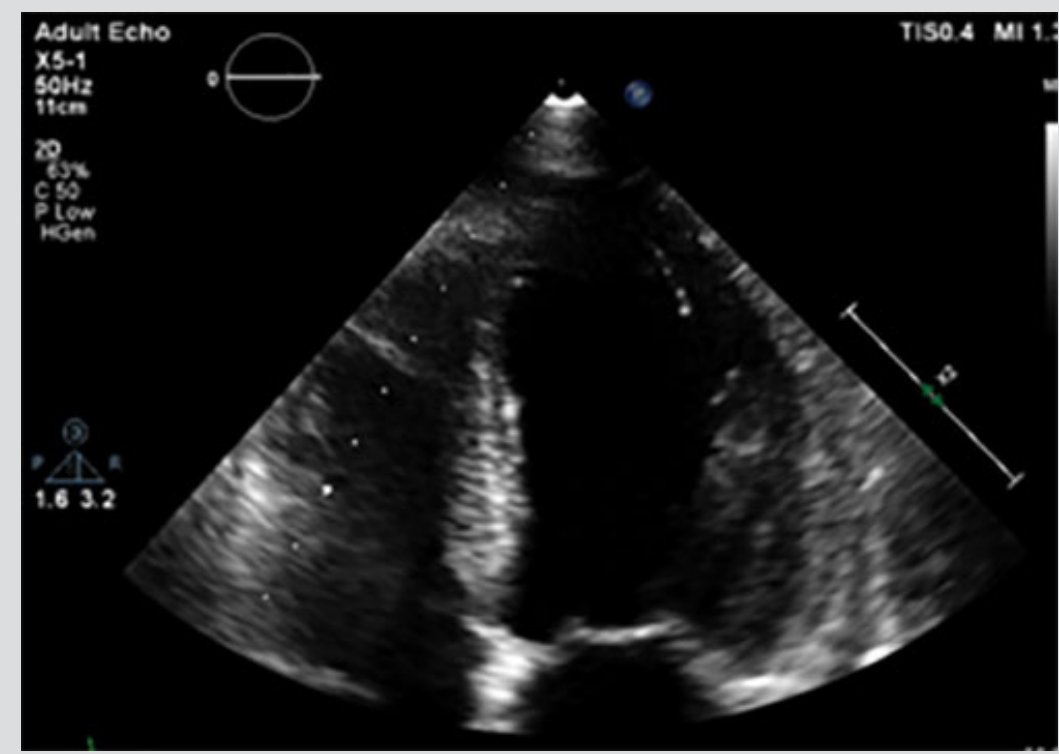

Figure 2.Cardiac ultrasonography showing diffuse septo-apical akinesia with global ejection fraction impairment (4-chambers view). 


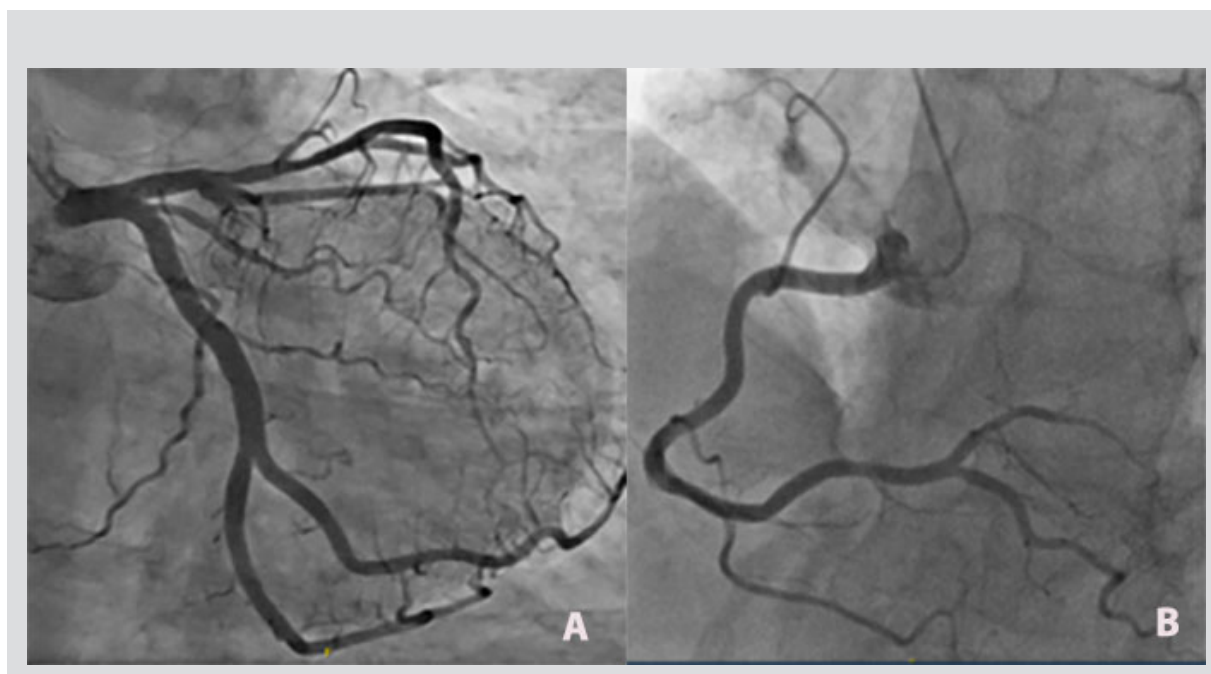

Figure 3 (a). Selective left coronary angiogram showing no significant lesions.

(b). Selective right coronary angiogram showing no significant lesions.

Therewas no recurrence of either the hyponatremia or the hypokalaemia, which were probablycaused by the vomiting, or of hypomagnesemia, which resolved after supplementation and stopping the PPI. Hypocalcaemia was probably secondary to severe hypomagnesemia. Further investigations ruled out the presence of a pheochromocytoma.

\section{DISCUSSION}

Hyponatremia, hypokalaemia and hypomagnesemia are common abnormalities caused by heart failure, as electrolyte homeostasis is affected by the neurohormonal responses to low cardiac output and by medication for heart failure (in particular diuretic therapy).

Hyponatremia appears to be an independent predictor of increased mortality ${ }^{[1]}$, while hypokalaemia is correlated with sudden cardiac death ${ }^{[2]}$.

Reciprocally, cellular contraction mechanisms can be influenced by electrolyte abnormalities and impact on cardiac function and promote heart failure. Current heart failure guidelines do not highlight the importance of correcting hypomagnesemia and hypocalcaemia which possibly contribute to failure of the heart's pumping action.

Hypocalcaemia has been related to reversible heart failure which was treated with supplementation, although the mechanism is not well understood. A recent case report associated hypocalcaemia with diffuse TC-like akinesia ${ }^{[3]}$.

Recognised as a predisposing factor for malignant ventricular arrhythmias, hypomagnesemia has also been associated with the development of mitochondrial alterations leading to myocardiocyte death and hypercoagulability. Moreover, magnesium directly provokes myocardial vasodilation, indirectly affecting cardiac contractility ${ }^{[4]}$.

Our patient presented with new ECG abnormalities, troponin elevation, and wall motion abnormalities that extended beyond the territory of a single artery, without coronary artery occlusion. This clinical presentation corresponds with TC, which we attributed principally to electrolyte abnormalities.

The most common TC triggers described in the literature are emotional stress (fear, grief), neurological events (cerebrovascular accidents), invasive procedures, drug administration (dobutamine, SSRI) and, recently, electrolyte abnormalities.

Catecholamine-induced cardiotoxicity and microvasculature dysfunction are considered the most common causes of TC ${ }^{[5]}$.

The following points should be kept in mind:

- $\quad$ Electrolyte disorders (as reported for hypomagnesemia) may provoke the microvascular dysfunction believed to cause TC.

- $\quad$ A cellular mechanism related to electrolyte disorders can contribute to cardiac impairment, as can occur in hypocalcaemia.

- An electrical abnormality could induce a neurohormonal stress reaction, while a neurovegetative mechanism could provoke heart failure.

- $\quad$ Akinesia persists for a while after a trigger has been removed because of the phenomenon of myocardial stunning.

- $\quad$ TC describes a group of heterogeneous conditions whose physiopathology probably slightly differs from each other. 


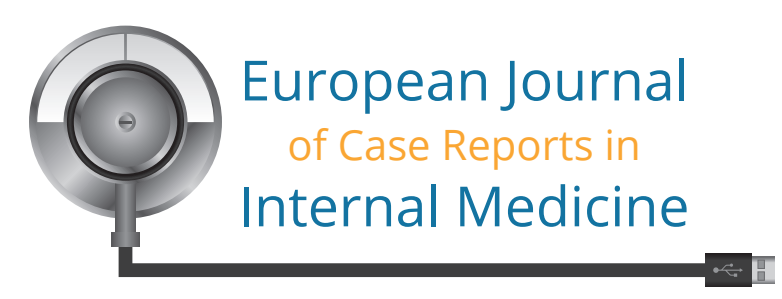

Further research into TC physiopathology, the impact of electrolytes on cellular function and the possible impact of cellular dysfunction on neurohormonal response is required.

This case report underlines the link between electrolyte disturbances and wall motion abnormalities beyond the well-known proarrhythmogenic effects of electrolyte abnormalities.

Consequently, in clinical practice, when heart failure is suspected, electrolyte abnormalities should be carefully ruled out because of their possible triggering role and, if present, should be corrected as an essential part of cardiac treatment.

\section{REFERENCES}

1. De Luca L, Klein L, Udelson JE, Orlandi C, Sardella G, Fedele F, et al. Hyponatremia in patients with heart failure. Am J Cardiol 2005;96:19L-23L.

2. Macdonald JE, Struthers AD. What is the optimal serum potassium level in cardiovascular patients? J Am Coll Cardiol 2004;43:155-161.

3. Solzbach U, Kitterer HR, Haas H. Reversible congestive heart failure in severe hypocalcemia. Herz 2010;35:507-510.

4. Chakraborti S, Chakraborti T, Mandal M, Mandal A, Das S, Ghosh S. Protective role of magnesium in cardiovascular diseases: a review. Mol Cell Biochem 2002;238:163-179.

5. Kazuo K, Miho F, Toshihiro I, Shinichi H, Tohru M. Takotsubo cardiomyopathy: pathophysiology, diagnosis and treatment. World J Cardiol 2014;6:602-609. 